\title{
Pharmacokinetic and exploratory exposure-response analysis of pertuzumab in patients with operable HER2-positive early breast cancer in the APHINITY study
}

\author{
Whitney P. Kirschbrown ${ }^{1}$ - Matts Kågedal ${ }^{1}$ - Bei Wang ${ }^{1} \cdot$ Lars Lindbom $^{2} \cdot$ Adam Knott $^{3} \cdot$ Rachelle Mack $^{3}$. \\ Sharareh Monemi ${ }^{1} \cdot$ Ihsan Nijem $^{1}$. Sandhya Girish ${ }^{1} \cdot$ Christie Freeman $^{4} \cdot$ Debora Fumagalli $^{5} \cdot$ Robin McConnell $^{6}$. \\ Guy Jerusalem ${ }^{7}$. Chris Twelves ${ }^{8}$. José Baselga ${ }^{9} \cdot$ Gunter von Minckwitz ${ }^{10}$. José Bines ${ }^{11}$. Amit Garg ${ }^{1}$
}

Received: 22 October 2018 / Accepted: 25 March 2019 / Published online: 11 April 2019

(c) The Author(s) 2019

\begin{abstract}
Purpose To characterize the pharmacokinetics (PK) of, and perform an exploratory exposure-response (E-R) analysis for, pertuzumab in patients with HER2-positive early breast cancer (EBC) within the APHINITY study (NCT01358877, BIG 4-11/BO25126/TOC4939G).

Methods A previously developed pertuzumab two-compartment linear population pharmacokinetic (popPK) model was subjected to external validation to examine appropriateness for describing pertuzumab concentrations from the APHINITY study. Pharmacokinetic drug-drug interactions (DDIs) between pertuzumab, trastuzumab, and chemotherapy were assessed by comparing observed serum or plasma $C_{\max }, C_{\min }$, and $\mathrm{AUC}_{\text {last }}$ geometric mean ratios with $90 \%$ CIs. Predictions of pertuzumab $C_{\mathrm{max}, \mathrm{ss}}, C_{\mathrm{min,ss}}$, and $\mathrm{AUC}_{\mathrm{ss}}$ were derived from individual parameter estimates and used in an exploratory E-R analysis.

Results Using data from 72 patients, based on goodness-of-fit, the popPK model was deemed appropriate for predictions of individual exposures for subsequent comparisons to historical data, assessment of DDIs, and E-R analyses. No evidence of DDIs for pertuzumab on trastuzumab, trastuzumab on pertuzumab, or pertuzumab on chemotherapy PK was observed. Analyses of differences in exposure between patients with and without invasive disease-free survival events did not indicate improved efficacy with increased exposure. Overall Grade $\geq 3$ diarrhea prevalence was higher with pertuzumab versus placebo, but was not greater with increasing pertuzumab exposure. No apparent E-R relationship was suggested with respect to other grade $\geq 3$ AEs.

Conclusion Overall, the limited available data from this exploratory study suggest that no dose adjustments are needed for pertuzumab when administered in combination with trastuzumab and an EBC chemotherapy regimen.
\end{abstract}

Keywords Pharmacokinetics $\cdot$ Exposure-response $\cdot$ Drug-drug interactions $\cdot$ Pertuzumab

\section{Introduction}

Pertuzumab (PERJETA ${ }^{\circledR}$, F. Hoffmann-La Roche Ltd, Basel, Switzerland) is a recombinant, humanized immunoglobulin G1 $\kappa$ monoclonal antibody that targets human epidermal growth factor receptor 2 (HER2), a transmembrane

Electronic supplementary material The online version of this article (https://doi.org/10.1007/s00280-019-03826-1) contains supplementary material, which is available to authorized users.

Amit Garg

garg.amit@gene.com

Extended author information available on the last page of the article glycoprotein with intrinsic tyrosine kinase activity [1]. By binding to extracellular subdomain 2, pertuzumab prevents dimerization of HER2 with other HER family receptors $[2,3]$. As a result, pertuzumab inhibits two major ligandinitiated intracellular signaling pathways, mitogen-activated protein kinase and phosphoinositide 3-kinase, thereby inducing cell growth arrest and apoptosis [4].

Pertuzumab and trastuzumab (Herceptin ${ }^{\circledR}$, F. HoffmannLa Roche Ltd) bind to different epitopes on the HER2 receptor and have distinct mechanisms for disrupting HER2 signaling. Due to their complementary modes of action, the combination of these two anti-HER2 antibodies provides more comprehensive HER2 pathway blockade than single agents $[5,6]$. Pertuzumab was first approved for use in 
combination with trastuzumab and docetaxel in patients with HER2-positive metastatic breast cancer (MBC), based on the pivotal phase III CLEOPATRA study (NCT00567190), which showed significant improvement in progression-free survival with the combination as compared with placebo plus trastuzumab and docetaxel [7].

Pertuzumab is also licensed as neoadjuvant treatment for patients with HER2-positive early breast cancer (EBC); approval was supported by two phase II studies, NeoSphere (NCT00545688) and TRYPHAENA (NCT00976989), in which the addition of pertuzumab to trastuzumab and chemotherapy significantly improved pathologic complete response rate (pCR), without increasing cardiac toxicities $[8,9]$.

The clinical pharmacokinetics (PK) of pertuzumab were first described in 481 patients with a variety of solid tumors from 11 phase I/II studies and CLEOPATRA [10]. A twocompartment linear model with first-order elimination was used to characterize pertuzumab PK in the $2-25 \mathrm{mg} / \mathrm{kg}$ dose range, a range which includes the approved fixed-dose regimen of an $840 \mathrm{mg}$ loading dose followed by a $420 \mathrm{mg}$ maintenance dose, administered intravenously on an every-3week ( $\mathrm{q} 3 \mathrm{w}$ ) schedule [10]. In the final model of this analysis, covariates explained $21.6 \%$ of the between-subject variability for clearance (CL) and $35.0 \%$ of the between-subject variability for volume of central compartment $\left(V_{\mathrm{c}}\right)$, with lean body weight (LBW) and serum albumin (ALBU) being identified as statistically significant covariates of pertuzumab PK [10]. LBW impacted pertuzumab PK: increased LBW correlated with increasing CL, $V_{\mathrm{c}}$, and volume of peripheral compartment $\left(V_{\mathrm{p}}\right)$ [10]. Sensitivity analyses demonstrated that the magnitude of its effect on the pertuzumab exposure measures minimum concentration at steady state $\left(C_{\text {min,ss }}\right)$, maximum concentration at steady state $\left(C_{\mathrm{max}, \mathrm{ss}}\right)$, and area under the concentration-time curve at steady state $\left(\mathrm{AUC}_{\mathrm{ss}}\right)$ was small relative to the overall between-subject variability in the population [10]. The authors concluded that, because of this, dose adjustment for LBW is not warranted [10].

The maximum tolerated dose for pertuzumab was not reached in clinical studies; therefore, the selected dose for CLEOPATRA and NeoSphere was based on achievement of a trough concentration at steady state $\left(C_{\text {trough,ss }}\right)$ of $\geq 20 \mu \mathrm{g} / \mathrm{mL}$ in $90 \%$ of patients (i.e., clinical target concentration) [11]. Non-clinical xenograft dose-response studies showed maximal suppression of tumor growth when the $C_{\text {trough }}$ was maintained above this threshold [12]. Over $90 \%$ of patients achieved the target serum pertuzumab concentration in the NeoSphere study, and the exposure-response analysis suggested that there was no association between $\mathrm{pCR}$ rate and pertuzumab concentrations within the observed concentration range of 20-100 $\mu \mathrm{g} /$ $\mathrm{mL}$ [11]. This analysis further supported the appropriateness of the fixed, non-weight-based pertuzumab dose of $840 \mathrm{mg}$ followed by $420 \mathrm{mg} \mathrm{q} 3 \mathrm{w}$ in the neoadjuvant treatment of patients with early breast cancer [11].

More recently, pertuzumab was approved for the adjuvant treatment of patients with HER2-positive EBC based on the APHINITY study (NCT01358877, BIG 4-11/BO25126/ TOC4939G). APHINITY was a prospective, randomized, multicenter, multinational, double-blind, placebo-controlled, phase III study that compared intravenous pertuzumab (18 cycles), trastuzumab (18 cycles), and chemotherapy (3-4 cycles of anthracycline-containing chemotherapy followed by 3-4 cycles of taxane-containing chemotherapy or 6 cycles of docetaxel plus carboplatin) with placebo, trastuzumab, and chemotherapy as adjuvant therapy in patients with operable HER2-positive early breast cancer (EBC) [13]. APHINITY met its primary objective, showing significantly improved rates of invasive disease-free survival (IDFS) with the addition of pertuzumab to trastuzumab and chemotherapy. A disease recurrence event occurred in $7.1 \%$ of patients treated with pertuzumab and in $8.7 \%$ of patients who received placebo (a difference of $1.6 \%$; hazard ratio $0.81 ; 95 \%$ confidence interval $[\mathrm{CI}] 0.66-1.00 ; p=0.045)$. In terms of safety, diarrhea (a common adverse event with pertuzumab) of Grade 3 or higher occurred more frequently with pertuzumab than with placebo, and was mostly experienced during chemotherapy [13].

Data described herein were collected as part of an optional APHINITY Global PK sub-study; designed to characterize pertuzumab steady-state pharmacokinetics in patients with HER2-positive EBC and to support the current pertuzumab dosing regimen in this population. The key objectives for this PK analysis were to: (1) characterize the steady-state PK of pertuzumab in patients with HER2-positive EBC; (2) characterize the potential PK drug-drug interactions (DDIs) between the therapeutic proteins trastuzumab and pertuzumab, and between pertuzumab and paclitaxel and carboplatin. In addition, three exploratory objectives addressed by this PK analysis were to: (1) compare the steady-state concentrations of pertuzumab, when administered as adjuvant treatment to patients with EBC, with data obtained previously in women with MBC (CLEOPATRA); (2) compare the steady-state concentrations of trastuzumab when administered as adjuvant treatment to patients with $\mathrm{EBC}$, with data obtained previously in women with EBC receiving adjuvant treatment (HERA [NCT00045032]); (3) perform an exploratory analysis of exposure-response relationships, including IDFS and diarrhea as the key efficacy and safety endpoints, respectively. 


\section{Materials and methods}

\section{APHINITY Global PK sub-study design}

The design of the APHINITY study has been reported previously [13]. APHINITY included an optional Global PK sub-study with a separate protocol from the main trial.

\section{PK sampling}

PK samples were collected only in the PK sub-study. A sparse PK sampling approach was adopted. The PK sampling times for pertuzumab and trastuzumab were pre- and post-dose on cycles 1, 10, and 15 (and Cycle 2 pre-dose). PK sampling times for chemotherapy included 3 , 5 , and $24 \mathrm{~h}$ post-dose for paclitaxel, 3 and $5 \mathrm{~h}$ post-dose for the metabolite 6-alpha-hydroxy paclitaxel, and 1, 2, 4, and $5 \mathrm{~h}$ post-dose for carboplatin.

\section{Bioanalytical methods}

Validated assays were used to measure pertuzumab, trastuzumab, paclitaxel and 6-alpha-hydroxy paclitaxel, and carboplatin from blood samples.

The serum concentrations of pertuzumab were determined by an enzyme-linked immunosorbent assay described previously [14]. The assay used a monoclonal anti-idiotype antibody against pertuzumab to capture pertuzumab from serum samples. Bound pertuzumab was detected with a biotinylated monoclonal antibody (10C4; Antibody Engineering, Genentech, Inc., South San Francisco, CA, USA) against a Genentech, Inc. immunoglobulin G framework and horseradish peroxidase-Avidin D conjugate. A peroxidase substrate (tetramethyl benzidine) was used for color development to quantify serum pertuzumab against a standard curve. The lower limit of quantification in human serum was $150 \mathrm{ng} / \mathrm{mL}$ with a standard curve reporting range of $150-4000 \mathrm{ng} / \mathrm{mL}$ (limit of detection was $62.5 \mathrm{ng} / \mathrm{mL}$ ). The inter-assay accuracy (percentage difference) ranged from -8.75 to $3.84 \%$ while the inter-assay precision (percentage coefficient of variation) ranged from 3.89 to $15.3 \%$. The presence of trastuzumab did not interfere with the accurate quantification of pertuzumab in this assay.

Trastuzumab serum concentrations were determined by a validated high-performance liquid chromatography with tandem mass spectrometry (LC-MS/MS) detection described previously [15]. An affinity capture approach using streptavidin magnetic beads coupled with biotinylated recombinant human HER2 extracellular domain was used to enrich trastuzumab from human serum. The bound trastuzumab protein was subjected to 'on-bead' proteolysis with trypsin, following standard protein denaturation, reduction, and alkylation processing steps. Prior to digestion completion, working internal standard solution was added. The characteristic peptide fragments produced by this procedure were then quantified as surrogates of the total antibody concentration originating from trastuzumab by LC-MS/MS (i.e., multiple reaction monitoring [MRM]). The lower limit of quantification in human serum was $100 \mathrm{ng} / \mathrm{mL}$ with a standard curve reporting range of $100 \mathrm{ng} / \mathrm{mL}$ to $2500 \mathrm{ng} / \mathrm{mL}$. The interassay accuracy (percentage difference) ranged from -8.08 to $-1.47 \%$, while the inter-assay precision (percentage coefficient of variation) ranged from 3.07 to $8.44 \%$. The presence of pertuzumab did not interfere with the accurate quantification of trastuzumab in this assay.

Plasma concentrations of paclitaxel and its metabolite 6-alpha-hydroxy paclitaxel were determined by a validated liquid chromatography tandem mass spectrometry method. An aliquot of $50 \mu \mathrm{L}$ of human plasma (K $\mathrm{K}_{2}$ EDTA) sample containing paclitaxel and 6-alpha-hydroxy paclitaxel was extracted using supported-liquid extraction. The API 5000 Triple Quad ${ }^{\mathrm{TM}}$ (Applied Biosystems, Foster City, CA, USA) was operated in MRM mode under optimized conditions for the detection of paclitaxel and 6-alphahydroxy paclitaxel positive ions formed by electrospray ionization. Paclitaxel- $\mathrm{d}_{5}$ was used as an internal standard. Paclitaxel concentrations were calculated with the use of a standard curve with a $1 / x^{2}$ linear regression over a concentration range of $2.00-2500 \mathrm{ng} / \mathrm{mL}$. Concentrations of 6-alpha-hydroxy paclitaxel were calculated using a separate standard curve with a $1 / x^{2}$ linear regression over the same concentration range of $2.00-2500 \mathrm{ng} / \mathrm{mL}$. The interassay relative standard deviation ranged from 1.5 to $9.6 \%$ for paclitaxel and from 2.2 to $8.9 \%$ for 6-alpha-hydroxy paclitaxel. The inter-assay accuracy ranged from 86.0 to 96.6\% of nominal for paclitaxel and from 96.5 to $103.2 \%$ of nominal for 6-alpha-hydroxy paclitaxel. Stability of paclitaxel and 6-alpha-hydroxy paclitaxel was established in human plasma for 449 days at $-20{ }^{\circ} \mathrm{C}$ and 1280 days at $-70{ }^{\circ} \mathrm{C}$.

Carboplatin plasma concentrations were determined by a validated inductively coupled plasma tandem mass spectrometry method. Human plasma $\left(\mathrm{K}_{2}\right.$ EDTA) samples $(50 \mu \mathrm{L})$ containing carboplatin were analyzed on a PerkinElmer ELAN DRC II mass spectrometer optimized for the detection of platinum from carboplatin. Terbium was used as an internal standard. Platinum concentrations were calculated with the use of a standard curve with a $1 / x^{2}$ linear regression over a concentration range of $2.00-1000 \mathrm{ng} / \mathrm{mL}$. The inter-assay relative standard deviation ranged from 0.68 to $4.06 \%$, while the inter-assay accuracy ranged from 97.6 to $100.8 \%$ of nominal. Stability of platinum 
was established in human plasma for 195 days at $-20^{\circ} \mathrm{C}$ and $-70{ }^{\circ} \mathrm{C}$.

\section{Data handling}

Patients were defined as evaluable for pharmacokinetic (PK) analysis if they had at least one documented pertuzumab administration and a corresponding post-dose pertuzumab PK sample collection. Records were excluded if the time of drug administration or sample collection was missing. No imputation of PK values was performed. Observations with missing PK or time values, or those below the minimum quantifiable concentration, were omitted from the analysis.

Outliers were identified by visual inspection of each individual's concentration versus time profile. Typically, a data point was deemed an outlier if a trough concentration was greater than the peak concentration, or if the absolute residual variability was five times larger than the expected residual standard deviation.

\section{PK analysis}

Pertuzumab concentrations from the APHINITY Global PK sub-study were compared with predictions based on a previously developed pertuzumab population PK model [10]. This model was built on data collected from patients with solid tumors, including MBC, during five phase $\mathrm{I} / \mathrm{Ib}$ studies, six phase II studies, and one pivotal phase III study [10]. Most of the data (>95\%) used for population PK development were based on pertuzumab without concomitant trastuzumab treatment; seven of the 12 studies included investigated pertuzumab as a monotherapy. In the previously developed model, pertuzumab PK were described by a two-compartment linear model with a CL, central volume of distribution, and terminal elimination half-life of 0.235 L/day, $3.11 \mathrm{~L}$, and 18 days, respectively. The covariates identified as significantly influencing pertuzumab CL were baseline serum albumin and LBW, with $15.5 \%$ and $4.1 \%$ of the between-subject variability in CL explained by serum albumin and LBW, respectively.

Comparisons of pertuzumab concentrations from the APHINITY Global PK sub-study to the previously developed model predictions were performed using NONMEM version 7.3 software (ICON Development Solutions, Ellicott City, MD, USA). Post-processing of NONMEM analysis results was carried out in R version 3.2.2 (R Development Core Team, R: A language and environment for statistical computing. R Foundation for Statistical Computing, Vienna, Austria; ISBN 3-900051-07-0; URL https://www.R-proje ct.org/). Individual PK parameters were estimated using first-order conditional estimation with interaction.

To evaluate the agreement of the observed PK data in the APHINITY Global PK sub-study with the historical PK data based on the population PK model, a visual predictive check was performed. Pertuzumab serum concentrations for 10000 subjects were simulated using LBW resampled from the observed LBW in the pertuzumab arm as well as nominal dose times and amounts for each patient. Albumin levels were not measured in APHINITY. Therefore, the median observed baseline albumin level of $4.3 \mathrm{~g} / \mathrm{dL}$ (range 3.3-5.7 g/dL, $N=258$ ) in HannaH (NCT00950300), a study of subcutaneous or intravenous trastuzumab for EBC [16], was added as the value for those in the APHINITY Global PK sub-study. In the NeoSphere study, the median observed baseline albumin level was $4.4 \mathrm{~g} / \mathrm{dL}$ (range $3.1-5.3 \mathrm{~g} / \mathrm{dL}$, $N=180$ ), indicating that the selected median baseline albumin value of $4.3 \mathrm{~g} / \mathrm{dL}$ is appropriate for an EBC patient population and can be used to describe pertuzumab PK in the APHINITY study. Median predicted pertuzumab concentrations and a $95 \%$ prediction interval were compared with the observed data.

For the purpose of the exploratory exposure-response analysis, individually predicted pertuzumab serum concentrations based on each patient's observed serum concentrations and covariates were obtained. The predictions were derived by fixing the parameters in the structural and variance model to the parameter estimates in the historical validated population PK model and generating the individual empirical Bayes estimates with NONMEM by setting MAXEVAL $=0$. Individual exposure estimates (AUC, $C_{\text {min }}$, and $C_{\max }$ ) were subsequently obtained for use in the exposure-response analysis (detailed below). Diagnostic plots of observed data versus population prediction and individual prediction were examined for adequate fit. Plots of conditional weighted residual versus population prediction and versus time (after first and last doses) were inspected for evidence of systematic lack of fit, and to confirm the absence of bias in the error distributions.

\section{DDIs}

The DDI analysis was carried out using R version 3.2.2.

The potential effect of pertuzumab on the steady-state PK of trastuzumab was assessed by comparing the arithmetic means of serum trastuzumab concentrations at pre-dose $\left(C_{\mathrm{min}, \mathrm{ss}}\right)$ and post-infusion $\left(C_{\mathrm{max}, \mathrm{ss}}\right)$ in cycles 10 and 15 in the pertuzumab and placebo arms. In addition, the $90 \%$ CIs in the ratio of the geometric means (calculated by standard methods) were constructed. Similarly, the potential effect of pertuzumab on the PK of paclitaxel (and 6-alpha-hydroxy paclitaxel) and carboplatin was assessed by comparing the arithmetic means of $C_{\max }$ and area under the concentration-time curve over all concentration measurements $\left(\mathrm{AUC}_{\text {last }}\right)$ in Cycle 1 in the pertuzumab and placebo arms.

For paclitaxel and carboplatin, collection of multiple blood samples on Cycle 1 Day 1 allowed characterization 
of the post-infusion concentration-time curves using noncompartmental methods. $C_{\max }$ was defined as the maximum observed concentration and $\mathrm{AUC}_{\text {last }}$ was calculated using the linear trapezoidal rule and nominal observation times. The $90 \%$ CIs in the ratio of the geometric means were also constructed. All observations for 6-alpha-hydroxy paclitaxel at $24 \mathrm{~h}$ post-dose were reported as below the quantification limit. Thus, $\mathrm{AUC}_{\text {last }}$ was not calculated.

The potential effect of trastuzumab on the PK of pertuzumab was assessed by comparing pertuzumab $C_{\max }$ and $C_{\min }$ observed in the APHINITY Global PK sub-study with the predictions based on the population PK model. An adequate prediction of the observed PK by the historical model would suggest that there was no impact of trastuzumab on the PK of pertuzumab.

\section{Exploratory exposure-response analysis}

To generate individual pertuzumab exposure for patients in the pertuzumab arm, a simulation dataset was constructed based on estimated individual parameters for the population PK model, corresponding LBW and median albumin incorporated as described previously. For all patients with at least one available valid post-treatment concentration measurement of pertuzumab, predictions of $C_{\mathrm{max}, \mathrm{ss}}, C_{\mathrm{min}, \mathrm{ss}}$, and AUC at steady state $\left(\mathrm{AUC}_{\mathrm{ss}}\right)$ were derived. The simulation dataset consisted of a loading dose and three maintenance doses. The loading dose and infusion duration were taken from the NONMEM dataset for each patient in the pertuzumab arm; thereafter, three doses of $420 \mathrm{mg}$ pertuzumab were administered using a 30-min infusion with 3 weeks' dosing interval. Pertuzumab approximate steady state is achieved following the first maintenance dose, and therefore three maintenance doses were selected to ensure steady state across patients. Individual predictions of concentration at the end of infusion $\left(C_{\text {max,ss }}\right)$ and before next dose $\left(C_{\text {min,ss }}\right)$ were generated following the third maintenance dose for all patients. $\mathrm{AUC}_{\mathrm{ss}}$ was calculated by dividing the maintenance dose by individual clearance values.

The efficacy endpoint in the exposure-efficacy analysis was the primary study endpoint, IDFS [13]. IDFS is the time from randomization to recurrence of ipsilateral invasive breast tumor, recurrence of ipsilateral locoregional invasive disease, a distant disease recurrence, contralateral invasive breast cancer, or death from any cause. Patients who had not had an event at the time of data analysis were censored at the date they were last known to be event-free. The primary exposure metrics used in the exposure-efficacy analysis were individual predicted $C_{\text {min,ss }}$ and $\mathrm{AUC}_{\mathrm{ss}}$. Box plots were created to compare exposure $\left(C_{\text {min,ss }}\right.$ and $\left.\mathrm{AUC}_{\mathrm{ss}}\right)$ in the subset of patients with PK data who had an IDFS event $(N=4)$ versus the patients with PK data who did not $(N=31)$.
Adverse events (AEs) were considered for the analysis if there was a difference of $\geq 5 \%$ in the incidence of Grade $\geq 3$ AEs between the pertuzumab and placebo arms in the primary analysis [13]. Furthermore, to be considered for the analysis, pertuzumab PK data were required for $\geq 5$ patients experiencing the $\mathrm{AE}$; "any grade $\geq 3 \mathrm{AE}$ " (incidence $64.2 \%$ in the pertuzumab arm and $57.3 \%$ in the placebo arm in the overall population [13]) and Grade $\geq 3$ diarrhea (incidence $9.8 \%$ in the pertuzumab arm and $3.7 \%$ in the placebo arm in the overall population [13]) met these criteria. These safety endpoints were assessed as binary variables. Results were deemed exploratory and did not reflect formal statistical hypothesis testing.

\section{Results}

\section{Patients and samples}

Seventy-two patients consented to the optional APHINITY PK Global sub-study. Of these, 38 patients received pertuzumab, and 35 contributed at least one pertuzumab PK sample. The total number of pertuzumab PK observations was 163 . In addition, 36 patients in the pertuzumab arm and 34 in the placebo arm contributed at least one trastuzumab PK sample. The total number of trastuzumab PK observations was 339 (179 from the pertuzumab arm and 160 from the placebo arm).

Patient demographics are summarized in Online Resource 1. In the APHINITY Global PK sub-study, all 38 patients who received pertuzumab were female, with a median age of 51.5 years and a median LBW of $44.7 \mathrm{~kg}$. These demographic characteristics, as well as nodal status and hormone receptor status, were comparable between the APHINITY Global PK sub-study and APHINITY intention-to-treat populations [13].

\section{Pertuzumab PK}

The visual predictive check of the population PK model for the Global PK sub-study data is shown in Fig. 1. Overall, the population PK model predicted the serum concentrations reasonably well across cycles. $C_{\min }$ appeared to increase in later cycles, although the majority of the observed pertuzumab concentrations were within the $95 \%$ prediction interval. Graphical assessment of the match between observed and model-predicted pertuzumab concentrations (Fig. 2), as well as of conditional weighted residuals (Online Resource 2 ), suggested that the previous model could predict the observed data reasonably well. However, the observed trough concentrations at later cycles were slightly higher than predicted by the model. Based on goodness of fit, the 


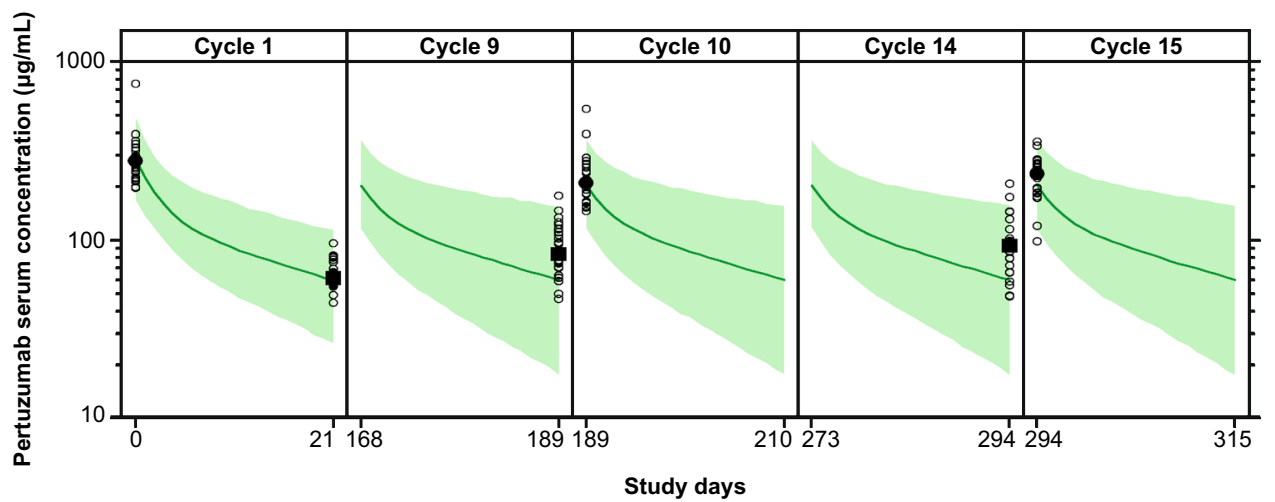

Fig. 1 Visual predictive check of pertuzumab concentrations. The open circles represent observed serum concentrations. The filled circles represent median observed $C_{\max }$. The filled squares represent median observed $C_{\min }$. The solid green line represents pre- dicted median serum concentration. The shaded area represents $95 \%$ prediction interval. $C_{\max }$ is maximum serum concentration. $C_{\min }$ is minimum serum concentration
Fig. 2 Observed versus modelpredicted pertuzumab serum concentrations. The solid black line represents line of identity. The solid green line and shaded green area are Gaussian Loess smooth with $95 \%$ confidence interval. The left-panel circles are individual observations/ population predictions. The right-panel circles are individual observations/individual predictions
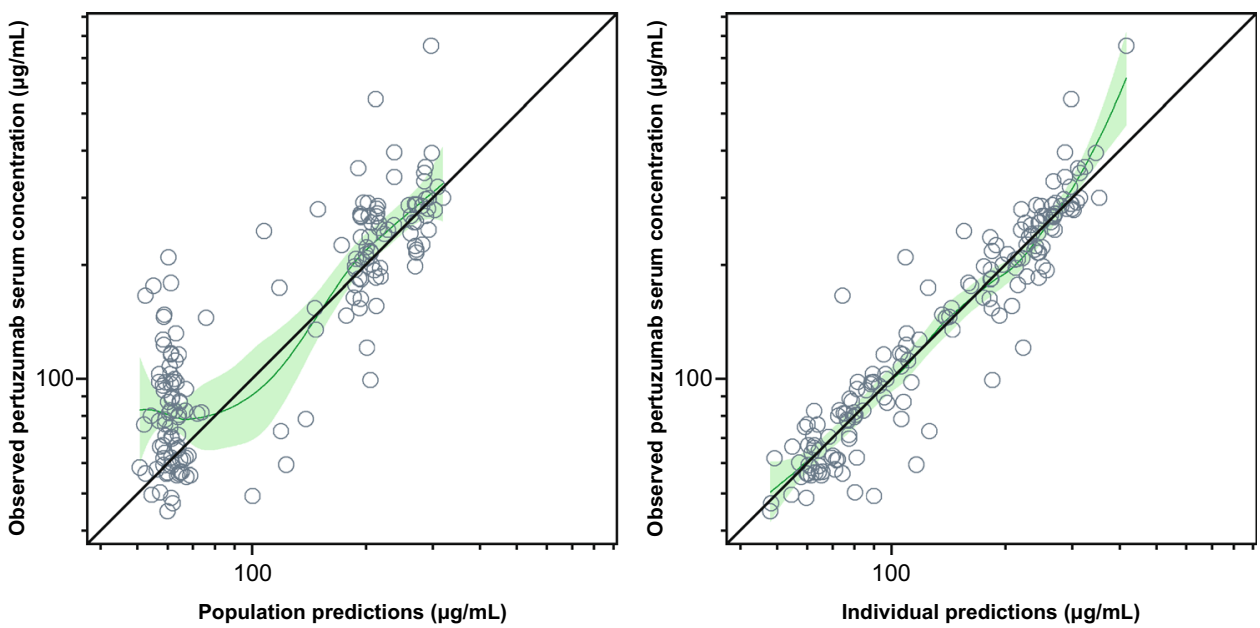

model was deemed appropriate for predictions of individual exposures for subsequent comparisons with historical data, assessment of potential DDIs, and exposure-response analyses.

\section{DDIs}

The $C_{\min }$ and $C_{\max }$ of trastuzumab with pertuzumab or placebo across cycles are shown and summarized in Fig. 3 and Online Resource 3, respectively. Trastuzumab geometric mean ratios were approximately $0.8-1.0$ and the $90 \%$ CIs all overlapped 1.0, indicating no impact of pertuzumab on trastuzumab serum $C_{\min }$ or $C_{\max }$ when administered in combination with an EBC chemotherapy regimen. There was also no evidence to suggest an impact of pertuzumab (in combination with trastuzumab) on $\mathrm{PK}$ of paclitaxel $\left(C_{\max }\right.$ and AUC), 6-alpha-hydroxy paclitaxel ( $C_{\max }$ only, as the observations at $24 \mathrm{~h}$ after dose were below the quantification limit) or carboplatin ( $C_{\max }$ and AUC). The data are shown in Online Resources 4, 5, and 6, respectively. It should be noted that carboplatin PK data were derived from only six patients in the pertuzumab arm and 12 patients in the placebo arm. An adequate prediction of observed pertuzumab PK in APHINITY by the historical model as described above suggested that there was no impact of trastuzumab on the PK of pertuzumab.

\section{Pertuzumab and trastuzumab exposure in EBC and MBC studies}

Serum pertuzumab $C_{\min }$ and $C_{\max }$ data in the APHINITY Global PK sub-study (cycles 1, 10, and 15) were comparable to PK data from the CLEOPATRA study in MBC (cycles 3, 9 , and 15) as shown in Table 1. 
Fig. 3 Trastuzumab $C_{\text {max }}$ and $C_{\min }$ with or without pertuzumab. The closed circles represent trastuzumab in the treatment arm (pertuzumab, trastuzumab, and chemotherapy). The open circles represent trastuzumab in the control arm (placebo, trastuzumab, and chemotherapy). The solid green line represents arithmetic mean for each timepoint and treatment arm. The shaded area is arithmetic mean \pm 1 standard deviation. $C_{\max }$ is maximum serum concentration. $C_{\min }$ is minimum serum concentration

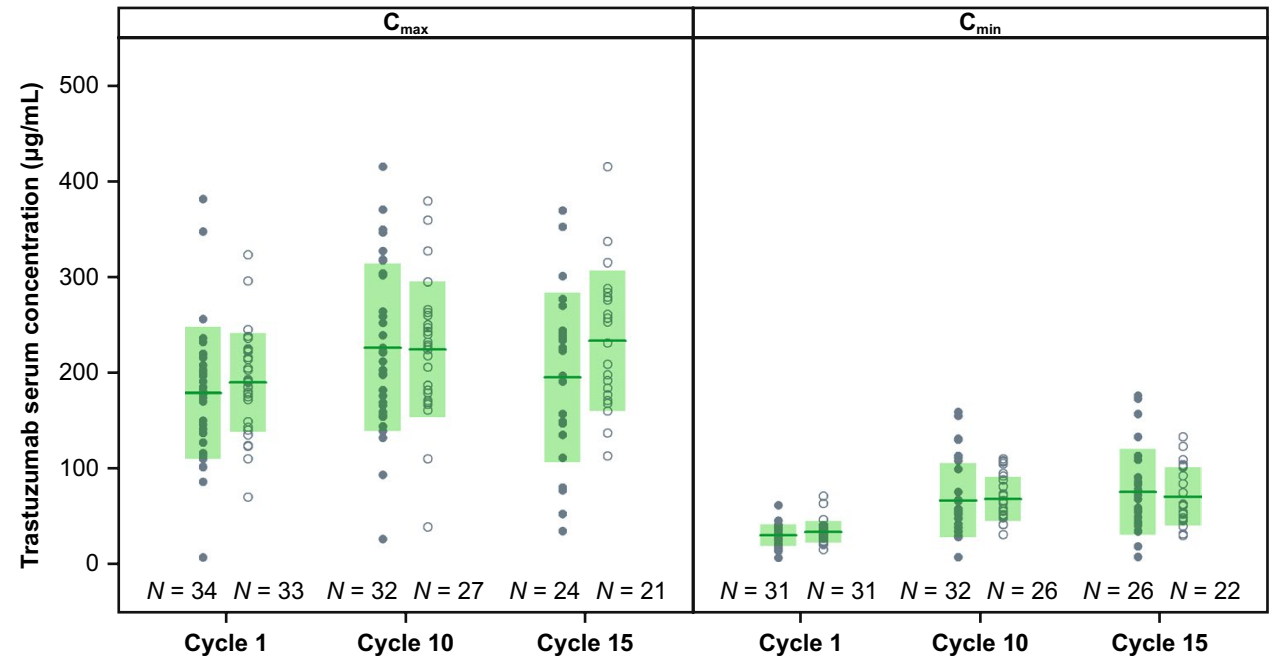

Table 1 Summary of $C_{\min }$ and $C_{\max }$ of pertuzumab in the presence of trastuzumab with and without chemotherapy (upper panel), and trastuzumab (lower panel) in patients with early breast cancer or metastatic breast cancer

\begin{tabular}{|c|c|c|c|c|c|c|c|c|c|}
\hline \multicolumn{10}{|c|}{ Pertuzumab } \\
\hline \multicolumn{5}{|c|}{ APHINITY (EBC) } & \multicolumn{5}{|c|}{ CLEOPATRA $(\mathrm{MBC})^{\mathrm{a}}$} \\
\hline Cycle & $n$ & $C_{\min }(\mu \mathrm{g} / \mathrm{mL})$ & $n$ & $C_{\max }(\mu \mathrm{g} / \mathrm{mL})$ & Cycle & $n$ & $C_{\min }(\mu \mathrm{g} / \mathrm{mL})$ & $n$ & $C_{\max }(\mu \mathrm{g} / \mathrm{mL})$ \\
\hline 1 & 30 & $65.9(12)$ & 30 & $291.2(99)$ & 3 & 18 & $63.4(48)$ & 18 & $183.4(34)$ \\
\hline 10 & 30 & $91.0(31)$ & 28 & $229.8(83)$ & 9 & 16 & $75.5(22)$ & 14 & $196.3(66)$ \\
\hline 15 & 24 & $98.4(40)$ & 21 & $232.8(65)$ & 15 & 11 & $94.1(31)$ & 9 & $221.1(32)$ \\
\hline
\end{tabular}

Trastuzumab

\begin{tabular}{|c|c|c|c|c|c|c|c|c|c|}
\hline \multicolumn{5}{|c|}{ APHINITY (EBC) } & \multicolumn{5}{|c|}{$\operatorname{HERA}(\mathrm{EBC})^{\mathrm{a}}$} \\
\hline Cycle & $n$ & $C_{\min }(\mu \mathrm{g} / \mathrm{mL})$ & $n$ & $C_{\max }(\mu \mathrm{g} / \mathrm{mL})$ & Cycle & $n$ & $C_{\min }(\mu \mathrm{g} / \mathrm{mL})$ & $n$ & $C_{\max }(\mu \mathrm{g} / \mathrm{mL})$ \\
\hline 10 & 58 & $67.7(32)$ & 59 & $225.5(80)$ & 10 & 3 & $66.0(39)$ & 3 & $203(7)$ \\
\hline 15 & 48 & $73.6(38)$ & 45 & $213.0(83)$ & 12 & 15 & $72.3(46)$ & 15 & $237(12)$ \\
\hline
\end{tabular}

Arithmetic means (SD)

$C_{\max }$ is maximum serum concentration, $C_{\min }$ is minimum serum concentration, $E B C$ is early breast cancer, $M B C$ is metastatic breast cancer, $S D$ is standard deviation

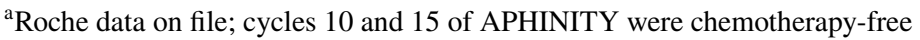

Serum trastuzumab $C_{\min }$ and $C_{\max }$ data at later cycles in the APHINITY Global PK sub-study (cycles 10 and 15) were comparable to observed trastuzumab PK data from the experimental arm of the HERA study in EBC (cycles 10 and 12), as demonstrated in Table 1. It should be noted that the observed trastuzumab data from APHINITY were pooled from pertuzumab and placebo arms, after confirming no PK DDI between pertuzumab and trastuzumab in the APHINITY Global PK sub-study.

\section{Exposure-efficacy analysis}

Thirty-five patients in the pertuzumab arm with predicted $\mathrm{AUC}_{\mathrm{ss}}$ and $C_{\mathrm{min}, \mathrm{ss}}$ were included in the exploratory exposure-efficacy analysis. There was no indication of improved efficacy, as defined by IDFS, with higher exposure to pertuzumab $\left(\mathrm{AUC}_{\mathrm{ss}}\right.$ and $C_{\mathrm{min}, \mathrm{ss}}$; Fig. $\left.4 \mathrm{a}\right)$. However, it should be noted that only four IDFS events occurred in the PK sub-study population, so there were limited data available for the analysis. 
Fig. 4 The relationships between pertuzumab exposure and efficacy or safety. Pertuzumab $\mathrm{AUC}_{\mathrm{ss}}, C_{\mathrm{min}, \mathrm{ss}}$, or $C_{\mathrm{max}, \mathrm{ss}}$ for patients in the pertuzumab arm with or without invasive disease-free survival (IDFS) event (a), any grade $\geq 3$ adverse event (AE) (b) or any grade $\geq 3$ diarrhea (c). The solid green line represents arithmetic mean for each group. The shaded area represents arithmetic mean \pm 1 standard deviation. $A U C_{s s}$ is area under the concentration-time curve at steady state. $C_{\max , s s}$ is maximum serum concentration at steady state. $C_{\min , s s}$ is minimum serum concentration at steady state
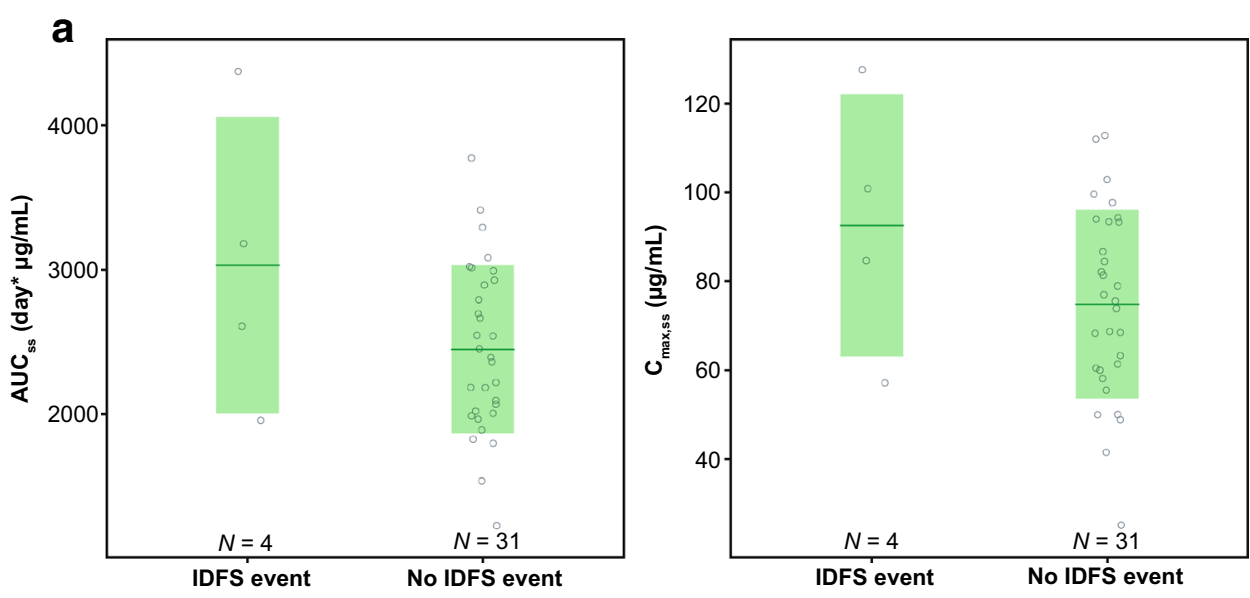

b
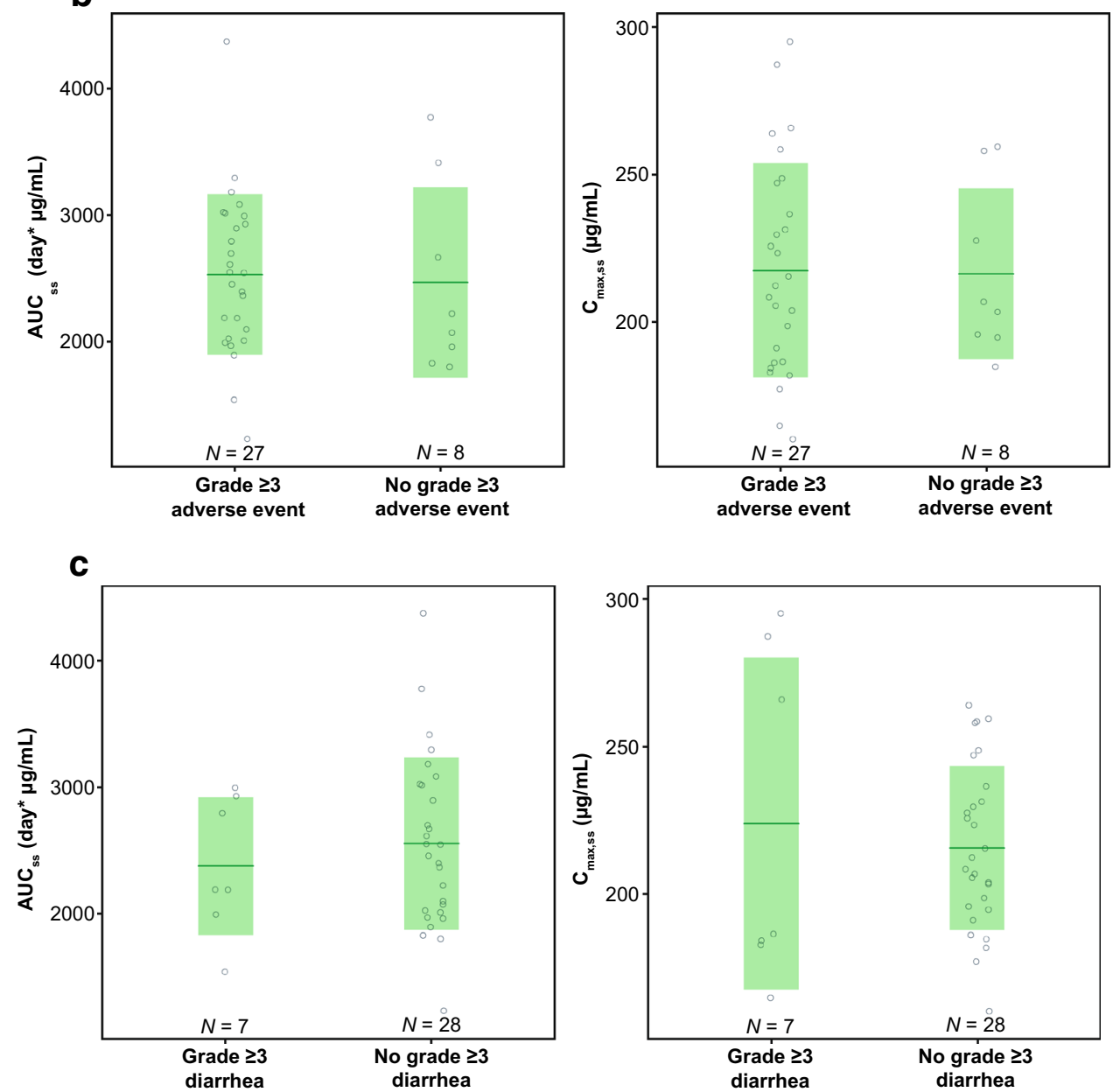

\section{Exposure-safety analysis}

In the safety dataset, 35 patients treated with pertuzumab had PK exposure predictions, and 34 patients in the placebo arm were used as a reference group to compare the potential exposure-response of pertuzumab. Table 2 summarizes the occurrence of Grade $\geq 3$ AEs for patients by treatment arm, as well as the patients treated with pertuzumab with 
Table 2 Grade $\geq 3$ AEs by PK group

\begin{tabular}{llll}
\hline Group & $n$ & Any grade $\geq 3 \mathrm{AE}^{\mathrm{a}}$ & Grade $\geq 3$ diarrhea \\
\hline Overall & & & \\
$\quad \begin{array}{l}\text { Placebo + trastuzumab + chemotherapy } \\
\text { Pertuzumab + trastuzumab + chemotherapy }\end{array}$ & 34 & $25(73.5)$ & $2(5.9)$ \\
AUC $_{\mathrm{ss}}$ & & $27(77.1)$ & $7(20.0)$ \\
$\quad$ Pertuzumab below the median & 18 & $13(72.2)$ & $4(22.2)$ \\
Pertuzumab above the median & 17 & $14(82.4)$ & $3(17.6)$ \\
$C_{\text {max,ss }}$ & & & $4(22.2)$ \\
Pertuzumab below the median & 18 & $13(72.2)$ & $3(17.6)$ \\
Pertuzumab above the median & 17 & $14(82.4)$ & \\
\hline
\end{tabular}

Data are patients, $n$ or patients, $n(\%)$

$A E$ is adverse event, $A U C$ is area under the concentration-time curve, $C_{\max }$ is maximum serum concentration, $P K$ is pharmacokinetic

${ }^{a}$ The most common Grade $\geq 3$ AEs were neutropenia, febrile neutropenia, neutrophil count decreased, diarrhea, and anemia [10] exposure $\left(\mathrm{AUC}_{\mathrm{ss}}\right.$ or $C_{\mathrm{max}, \mathrm{ss}}$ ) below or above median. The possible relationships between $\mathrm{AUC}_{\mathrm{ss}}$ or $C_{\mathrm{max}, \mathrm{ss}}$ and occurrence of any grade $\geq 3$ AEs are shown in Fig. 4b; the incidence of any grade $\geq 3$ AEs was similar in the pertuzumab and placebo arms, and the incidence of Grade $\geq 3$ AEs was similar in the high and low pertuzumab exposure groups (approximately 10\% difference but a difference of only one patient in the two groups).

The incidence of Grade $\geq 3$ diarrhea was more frequent in pertuzumab-treated patients than in placebo-treated patients, but the occurrence of Grade $\geq 3$ diarrhea did not increase with increasing pertuzumab exposure (Fig. 4c). It should be noted that grouping of patients based on $\mathrm{AUC}_{\mathrm{ss}}$ or $C_{\text {max,ss }}$ was identical, i.e., all patients with $\mathrm{AUC}_{\mathrm{ss}}$ above the median also had a $C_{\max , s s}$ above the median. Hence, results were identical for $C_{\text {max,ss }}$ and $\mathrm{AUC}_{\mathrm{ss}}$.

\section{Discussion}

The previously developed population PK model for pertuzumab, built on a large database of patients with MBC and other solid tumors [10], was used in the analyses of data collected from the APHINITY Global PK sub-study to characterize the PK of pertuzumab at steady state in the APHINITY patient population (EBC) and the potential interactions between pertuzumab and trastuzumab and chemotherapy. In addition, the steady-state concentrations of pertuzumab and trastuzumab were compared with historical data in patients with $\mathrm{EBC}$ or $\mathrm{MBC}$, and the exposure-response relationships were also explored.

The previously developed and validated population PK model adequately predicted pertuzumab serum concentrations across cycles in APHINITY. Although $C_{\text {min }}$ appeared to increase in later cycles, an increase of similar magnitude in pertuzumab $C_{\min }$ over time was also observed in CLEOPATRA and may be indicative of timedependent PK or treatment effect [14]. A definitive conclusion regarding time-dependency in pertuzumab PK cannot be made due to the small sample size in APHINITY. Also, it should be noted that clinical efficacy is expected if the target $C_{\text {trough }}$ for pertuzumab is maintained at or above $20 \mu \mathrm{g} / \mathrm{mL}$, and the increase in $C_{\min }$ had no apparent impact on safety [11]. While the observed $C_{\min }$ at later cycles tended to be slightly higher than predicted by the model, there was a large overlap and overall the data suggested no clinically meaningful difference among patients with EBC and other tumor types, including MBC (CLEOPATRA). This was further supported by the comparable observed PK of pertuzumab in the APHINITY and CLEOPATRA studies. Given that a population with EBC treated in the adjuvant setting has had surgical removal of tumor, when $\mathrm{PK}$ is found to be comparable to MBC and other solid tumor types, it suggests that tumor burden has no apparent impact on pertuzumab PK. Overall, the data demonstrated that this population PK model can be used for predictions of pertuzumab exposure in patients with HER2-positive EBC, in addition to other solid tumors included when the original model was developed.

Although most of the data used to build the population PK model were based on pertuzumab without concomitant trastuzumab, $C_{\min }$ and $C_{\max }$ of pertuzumab from patients in the APHINITY Global PK sub-study were adequately described by the model. In addition, the general agreement between observed pertuzumab concentrations and the values predicted by the population PK model suggested that trastuzumab had no clinically significant impact on the PK of pertuzumab in APHINITY.

Observed serum pertuzumab $C_{\min }$ and $C_{\max }$ data in APHINITY were comparable to pertuzumab $C_{\min }$ and $C_{\max }$ 
from the CLEOPATRA study in MBC at similar cycles. Additionally, serum trastuzumab $C_{\min }$ and $C_{\max }$ data in APHINITY were comparable to observed trastuzumab PK data from the experimental arm of the HERA study in EBC at similar cycles. These comparisons showed that pertuzumab PK data were comparable across different indications and chemotherapy combination partners and therefore indicates no impact of disease and chemotherapy on pertuzumab PK.

DDIs were not expected in this study, based on prior data $[11,14]$ and the distinct clearance mechanisms between monoclonal antibodies and the cytotoxic agents evaluated [17-19]. As expected, PK parameters $C_{\max }$ and $\mathrm{AUC}_{\text {last }}$ for paclitaxel and carboplatin were found to be similar in both treatment arms, indicating no impact of pertuzumab on the PK of these chemotherapeutic agents. The $90 \%$ CIs of the ratios of the PK parameters were wide and were most probably affected by the variability in PK parameters and the low statistical power associated with the small sample sizes between the treatment groups.

Similarly, no difference in trastuzumab PK parameters were observed in the presence of pertuzumab. PK exposure ratios (pertuzumab arm vs. placebo arm) were close to 1 at every time point, indicating no alteration in these values (Online Resource 3). No DDI between pertuzumab and trastuzumab was expected in this study as pertuzumab and trastuzumab bind to distinct epitopes of HER2 simultaneously without steric hindrance [20]. The $90 \%$ CIs for the ratios were large because of variability in PK parameters and the relatively small sample size. Collectively, the results demonstrate that the exposure of trastuzumab was not affected by pertuzumab administration in the presence of chemotherapy, confirming what was shown previously in the small CLEOPATRA DDI sub-study [14]). Overall, the data suggested that pertuzumab did not alter the PK of trastuzumab, paclitaxel, or carboplatin, and that pertuzumab PK was not altered by concurrent trastuzumab administration. DDIs between pertuzumab and trastuzumab and between pertuzumab and docetaxel were previously assessed in a small sub-study of the CLEOPATRA trial [14]; however, the sample size was relatively small $(n=17$ in the placebo arm and $n=20$ in the pertuzumab arm) and the current analysis helps to confirm the previous results.

Efficacy (based on IDFS) and safety (based on any grade $\geq 3 \mathrm{AE}$ and Grade $\geq 3$ diarrhea) were compared between high and low pertuzumab exposure groups. Only four IDFS events occurred in 35 patients with PK data. Analyses of differences in exposure $\left(\mathrm{AUC}_{\mathrm{ss}}\right.$ and $C_{\text {min,ss }}$ ) between patients with and without IDFS events did not indicate improved efficacy with higher pertuzumab exposure. While there was no indication of improved efficacy with higher pertuzumab exposure, the analysis is based on graphical assessment only and not a formal statistical test. Furthermore, the data were too limited to draw robust conclusions.

A difference in incidence of Grade $\geq 3$ AEs was observed between treatment arms in the overall APHINITY study (64.2\% in pertuzumab arm vs. $57.3 \%$ in placebo arm) [13]. This was also reflected in the APHINITY Global PK substudy populations in which 27 events were observed in 35 patients treated with pertuzumab, compared with 25 events in 34 patients treated with placebo. Analyses of differences in pertuzumab exposure (below or above median $C_{\text {max,ss }}$ and $\mathrm{AUC}_{\mathrm{ss}}$ ) in patients with any grade $\geq 3$ AEs showed a small difference between the exposure groups. Therefore, the available data did not suggest any exposure-safety relationship. It should, however, be noted that there was a limited number of patients in the pertuzumab-treated arm for robust statistical analyses.

A comparatively large difference in incidence of Grade $\geq 3$ diarrhea was observed between the two treatment groups ( $20 \%$ in pertuzumab arm vs. $5.9 \%$ in placebo arm). The difference was similar to that seen in the overall APHINITY study (9.8\% in pertuzumab arm vs. $3.7 \%$ in placebo arm). Analyses of differences in pertuzumab exposure (below or above median $C_{\mathrm{max}, \mathrm{ss}}$ and $\mathrm{AUC}_{\mathrm{ss}}$ ) did not indicate a higher incidence of Grade $\geq 3$ diarrhea with higher exposure to pertuzumab.

In this exploratory APHINITY Global PK sub-study, the PK of pertuzumab in patients with HER2-positive EBC were in line with predictions from a previously developed and validated population PK model [10]. These analyses demonstrate that no dose adjustments are necessary for pertuzumab and trastuzumab when the two monoclonal antibodies are administered together with chemotherapy (anthracyclineor non-anthracycline-containing) in patients with EBC. It should, however, be noted that data were limited due to small sample sizes, and by the fact that only four patients had an IDFS event. Overall, the limited available data from this exploratory study suggest that no dose adjustments are needed for pertuzumab when administered in combination with trastuzumab and an EBC chemotherapy regimen.

Acknowledgements The authors would like to thank: the patients and their families; the study partners: BIG, BrEAST, FSS, and F. Hoffmann-La Roche Ltd; the study sponsor: F. Hoffmann-La Roche Ltd; the Central Lab: IEO; the Steering Committee members; all members of the other committees involved in APHINITY: the Translational Advisory Committee, the Independent Data Monitoring Committee, the Interface Committee and the Cardiac Advisory Board. Support for third-party editorial assistance for this manuscript, furnished by Nantaporn Lertkowit, $\mathrm{PhD}$, of Health Interactions, was provided by $\mathrm{F}$. Hoffmann-La Roche Ltd, Basel, Switzerland.

Funding This study was funded by F. Hoffmann-La Roche Ltd, Basel, Switzerland. 


\section{Compliance with ethical standards}

Conflict of interest All authors received support for third-party writing assistance for this manuscript, provided by F. Hoffmann-La Roche Ltd. W.P.K. and A.G. are paid employees of Genentech, Inc., significant stockholders for Roche Holding Ltd, and inventors on a pertuzumab-related patent filing. M.K., B.W., I.N., S.M., and S.G. are paid employees of Genentech, Inc. and significant stockholders for Roche Holding Ltd. L.L. is a paid employee of, and has a significant ownership in, qPharmetra LLC. A.K. is a paid employee of Roche Products Limited and a significant stockholder for Roche Holding Ltd. R.Ma. was a paid employee of Roche Products Limited at the time of the study. D.F. received funding to run the conduct clinical trials from F. Hoffmann-La Roche Ltd/Genentech, Inc., AstraZeneca, Novartis, Servier, Tesaro, Pfizer, and GlaxoSmithKline, paid to her institution, and research grants from the charitable organizations BCRF, Foundation Cancer Luxembourg, and the Belgian National Lottery, paid to her institution. R.Mc. received funding to assist in the running of the APHINITY study from F. Hoffmann-La Roche Ltd, paid to his institution. G.J. has received honoraria from Novartis, Roche, Lilly, Pfizer, Amgen, and Bristol-Myers Squibb; holds a consulting/advisory role for Novartis, Celgene, Roche, Amgen, Pfizer, Bristol-Myers Squibb, Lilly, Puma Biotechnology, AstraZeneca, Daiichi Sankyo, and AbbVie; and has received research funding from Novartis and Roche. C.T. has received honoraria from Daiichi Sankyo, Roche, Pfizer, and Eisai, and travel expenses from MSD and Daiichi Sankyo. J.Ba. holds leadership positions in Varian Medical Systems, Bristol-Myers Squibb, Foghorn, Grail, Aura, and Infinity; consulting/advisory roles for Grail, PMV Pharma, ApoGen, Juno Therapeutics, Roche/Genentech, Lilly, Novartis, and Northern Biologics; stock or other ownership in PMV Pharma, Grail, Tango, Venthera, Juno, Varian, Foghorn, Aura, Infinity, ApoGen; and has received honoraria, travel, accommodations, and expenses from Roche/Genentech, Novartis, Lilly. G.v.M. has received research funding from Pfizer, Amgen, Celgene, AstraZeneca, Myriad Genetics, AbbVie, Vifor, and Roche, to his institution. J. Bi. has received clinical study funding from Roche as PI of the study; travel expenses to medical meetings from AstraZeneca and Roche; and holds consulting roles (advisory boards) with AbbVie, AstraZeneca, Genomic Health, Libbs, Pfizer, and Roche. C.F. declares no other conflict of interest.

Ethical approval The study was conducted in full accordance with the guidance of Good Clinical Practice guidelines and the Declaration of Helsinki. The protocol was approved by the institutional review board at each participating site. All participants provided written informed consent.

Data sharing statement Qualified researchers may request access to individual patient level data through the clinical study data request platform (https://www.clinicalstudydatarequest.com) after 31 July 2020. Before this date, qualified researchers may request access to individual patient level data by submitting, within a call for proposals, a Research Proposal to BIG. Further details on Roche's criteria for eligible studies are available here (https://clinicalstudydatarequest.com/Study-Spons ors/Study-Sponsors-Roche.aspx). For further details on Roche's Global Policy on the Sharing of Clinical Information and how to request access to related clinical study documents, see here (https://www.roche.com/ research_and_development/who_we_are_how_we_work/clinical_trial s/our_commitment_to_data_sharing.htm).

Open Access This article is distributed under the terms of the Creative Commons Attribution 4.0 International License (http://creativeco mmons.org/licenses/by/4.0/), which permits unrestricted use, distribution, and reproduction in any medium, provided you give appropriate credit to the original author(s) and the source, provide a link to the Creative Commons license, and indicate if changes were made.

\section{References}

1. Adams CW, Allison DE, Flagella K, Presta L, Clarke J, Dybdal N, McKeever K, Sliwkowski MX (2006) Humanization of a recombinant monoclonal antibody to produce a therapeutic HER dimerization inhibitor, pertuzumab. Cancer Immunol Immunother 55:717-727

2. Agus DB, Akita RW, Fox WD, Lewis GD, Higgins B, Pisacane PI, Lofgren JA, Tindell C, Evans DP, Maiese K, Scher HI, Sliwkowski MX (2002) Targeting ligand-activated ErbB2 signaling inhibits breast and prostate tumor growth. Cancer Cell 2:127-137

3. Diermeier-Daucher S, Hasmann M, Brockhoff G (2008) Flow cytometric FRET analysis of erbB receptor interaction on a cell-by-cell basis. Ann N Y Acad Sci 1130:280-286

4. Yarden Y, Sliwkowski MX (2001) Untangling the ErbB signalling network. Nat Rev Mol Cell Biol 2:127-137

5. Baselga J, Swain SM (2009) Novel anticancer targets: revisiting ERBB2 and discovering ERBB3. Nat Rev Cancer 9:463-475

6. Scheuer W, Friess T, Burtscher H, Bossenmaier B, Endl J, Hasmann M (2009) Strongly enhanced antitumor activity of trastuzumab and pertuzumab combination treatment on HER2-positive human xenograft tumor models. Cancer Res 69:9330-9336

7. Baselga J, Cortés J, Kim SB, Im SA, Hegg R, Im YH, Roman L, Pedrini JL, Pienkowski T, Knott A, Clark E, Benyunes MC, Ross G, Swain SM, CLEOPATRA Study Group (2012) Pertuzumab plus trastuzumab plus docetaxel for metastatic breast cancer. N Engl J Med 366:109-119

8. Gianni L, Pienkowski T, Im YH, Roman L, Tseng LM, Liu MC, Lluch A, Staroslawska E, Haba-Rodriguez J, Im SA, Pedrini JL, Poirier B, Morandi P, Semiglazov V, Srimuninnimit V, Bianchi G, Szado T, Ratnayake J, Ross G, Valagussa P (2012) Efficacy and safety of neoadjuvant pertuzumab and trastuzumab in women with locally advanced, inflammatory, or early HER2-positive breast cancer (NeoSphere): a randomised multicentre, open-label, phase 2 trial. Lancet Oncol 13:25-32

9. Schneeweiss A, Chia S, Hickish T, Harvey V, Eniu A, Hegg R, Tausch C, Seo JH, Tsai Y-, Ratnayake J, McNally V, Ross G, Cortés J (2013) Pertuzumab plus trastuzumab in combination with standard neoadjuvant anthracycline-containing and anthracycline-free chemotherapy regimens in patients with HER2-positive early breast cancer: a randomized phase II cardiac safety study (TRYPHAENA). Ann Oncol 24:2278-2284

10. Garg A, Quartino A, Li J, Jin J, Wada DR, Li H, Cortés J, McNally V, Ross G, Visich J, Lum B (2014) Population pharmacokinetic and covariate analysis of pertuzumab, a HER2-targeted monoclonal antibody, and evaluation of a fixed, non-weight-based dose in patients with a variety of solid tumors. Cancer Chemother Pharmacol 74:819-829

11. Quartino AL, Li H, Jin JY, Wada DR, Benyunes MC, McNally V, Viganò L, Nijem I, Lum BL, Garg A (2017) Pharmacokinetic and exposure-response analyses of pertuzumab in combination with trastuzumab and docetaxel during neoadjuvant treatment of HER2 early breast cancer. Cancer Chemother Pharmacol 79:353-361

12. Malik MA, Totpal K, Balter I, Sliwkowski MX, Pelletier N, Reich M, Crocker L, Friess T, Bauer S, Fiebig HH, Allison DE (2003) Dose response studies of recombinant humanized monoclonal antibody 2C4 in tumor xenograft models. Proc Am Assoc Cancer Res 44:150 (abstract 773)

13. von Minckwitz G, Procter M, de Azambuja E, Zardavas D, Benyunes M, Viale G, Suter T, Arahmani A, Rouchet N, Clark E, Knott A, Lang I, Levy C, Yardley DA, Bines J, Gelber RD, Piccart M, Baselga J, APHINITY Steering Committee Investigators (2017) Adjuvant pertuzumab and trastuzumab in early HER2-positive breast cancer. N Engl J Med 377:122-131 
14. Cortés J, Swain SM, Kudaba I, Hauschild M, Patel T, Grincuka E, Masuda N, McNally V, Ross G, Brewster M, Marier JF, Trinh MM, Garg A, Nijem I, Visich J, Lum BL, Baselga J (2013) Absence of pharmacokinetic drug-drug interaction of pertuzumab with trastuzumab and docetaxel. Anticancer Drugs 24:1084-1092

15. Liu L, Xu K, Li J, Maia M, Mathieu M, Elliott R, Yang J, Nijem I, Kaur S (2018) Optimizing hybrid LC-MS/MS binding conditions is critical: impact of biotransformation on quantification of trastuzumab. Bioanalysis 10:1819-1831

16. Quartino AL, Hillenbach C, Li J, Li H, Wada RD, Visich J, Li C, Heinzmann D, Jin JY, Lum BL (2016) Population pharmacokinetic and exposure-response analysis for trastuzumab administered using a subcutaneous "manual syringe" injection or intravenously in women with HER2-positive early breast cancer. Cancer Chemother Pharmacol 77:77-88

17. van der Vijgh WJF (1991) Clinical pharmacokinetics of carboplatin. Clin Pharmacokinet 21:242-261
18. Keizer RJ, Huitema AD, Schellens JH, Beijnen JH (2010) Clinical pharmacokinetics of therapeutic monoclonal antibodies. Clin Pharmacokinet 49:493-507

19. Sonnichsen DS, Relling MV (1994) Clinical pharmacokinetics of paclitaxel. Clin Pharmacokinet 27:256-269

20. Lu D, Burris HA 3rd, Wang B, Dees EC, Cortes J, Joshi A, Gupta M, Yi JH, Chu YW, Shih T, Fang L, Girish S (2012) Drug interaction potential of trastuzumab emtansine (T-DM1) combined with pertuzumab in patients with HER2-positive metastatic breast cancer. Curr Drug Metab 13:911-922

Publisher's Note Springer Nature remains neutral with regard to jurisdictional claims in published maps and institutional affiliations.

\section{Affiliations}

\section{Whitney P. Kirschbrown ${ }^{1} \cdot$ Matts Kågedal ${ }^{1}$ - Bei Wang ${ }^{1} \cdot$ Lars Lindbom $^{2} \cdot$ Adam Knott $^{3} \cdot$ Rachelle Mack $^{3}$. Sharareh Monemi ${ }^{1} \cdot$ Ihsan Nijem $^{1}$. Sandhya Girish ${ }^{1}$. Christie Freeman ${ }^{4} \cdot$ Debora Fumagalli $^{5} \cdot$ Robin McConnell $^{6}$. Guy Jerusalem ${ }^{7}$. Chris Twelves ${ }^{8} \cdot$ José Baselga $^{9} \cdot$ Gunter von Minckwitz ${ }^{10}$. José Bines ${ }^{11} \cdot$ Amit Garg $^{1}$}

1 Genentech, 1 DNA Way, South San Francisco, CA 94080, USA

2 qPharmetra, Hälsovägen 7, 14157 Huddinge, Sweden

3 Roche Products Limited, 6 Falcon Way, Shire Park, Welwyn Garden City AL7 1TW, UK

4 Breast European Adjuvant Study Team (BrEAST) Data Center, Institut Jules Bordet, Boulevard de Waterloo 121 (7th Floor), 1000 Brussels, Belgium

5 Breast International Group, Boulevard de Waterloo 76, 1000 Brussels, Belgium

6 Frontier Science (Scotland), Grampian View, Kincraig, Inverness-Shire PH21 1NA, UK
7 International Breast Cancer Study Group, CHU Liège and Liège University, Domaine Universitaire du Sart Tilman, B35, 4000 Liège, Belgium

8 University of Leeds and Leeds Teaching Hospitals Trust, St James's University Hospital, Beckett Street, Leeds LS9 7TF, UK

9 Executive Vice-President Research \& Development Oncology, AstraZeneca, 950 Wind River Ln, Gaithersburg, MD 20878, USA

10 German Breast Group, GBG Forschungs, Martin-Behaim-Str. 12, 63263 Neu-Isenburg, Germany

11 Instituto Nacional de Câncer, Praça Cruz Vermelha, 23-Centro, Rio de Janeiro 20230-130, Brazil 